\title{
Automated reduction of statistical errors in the estimated correlation function matrix for operational modal analysis
}

Tarpø, Marius; Friis, Tobias; Olsen, Peter; Juul, Martin; Georgakis, Christos ; Brincker, Rune

Published in:

Mechanical Systems and Signal Processing

Link to article, DOI:

10.1016/j.ymssp.2019.07.024

Publication date:

2019

Document Version

Peer reviewed version

Link back to DTU Orbit

Citation (APA):

Tarpø, M., Friis, T., Olsen, P., Juul, M., Georgakis, C., \& Brincker, R. (2019). Automated reduction of statistical errors in the estimated correlation function matrix for operational modal analysis. Mechanical Systems and Signal Processing, 132, 790-805. https://doi.org/10.1016/j.ymssp.2019.07.024

\section{General rights}

Copyright and moral rights for the publications made accessible in the public portal are retained by the authors and/or other copyright owners and it is a condition of accessing publications that users recognise and abide by the legal requirements associated with these rights.

- Users may download and print one copy of any publication from the public portal for the purpose of private study or research.

- You may not further distribute the material or use it for any profit-making activity or commercial gain

- You may freely distribute the URL identifying the publication in the public portal 


\title{
Automated Reduction of Statistical Errors in the Estimated Correlation Function Matrix for Operational Modal Analysis
}

\author{
Marius Tarp $\varnothing^{1, *}$, Tobias Friis ${ }^{\mathrm{b}}$, Peter Olsen $^{\mathrm{a}}$, Martin Juul ${ }^{\mathrm{c}}$, Christos Georgakis $^{\mathrm{a}}$, Rune Brincker ${ }^{\mathrm{b}}$ \\ ${ }^{a}$ Aarhus University, Department of Engineering, Inge Lehmanns Gade 10, Aarhus, Denmark \\ ${ }^{b}$ Technical University of Denmark, Department of Civil Engineering, Brovej B.118, Kgs. Lyngby, Denmark \\ ${ }^{c}$ Brincker 83 Georgakis, Inge Lehmanns Gade 10, Aarhus, Denmark
}

\begin{abstract}
In operational modal analysis, the correlation function matrix is treated as multiple free decays from which system parameters are extracted. The finite time length of the measured system response, however, introduces statistical errors into the estimated correlation function matrix. These errors cause both random and bias errors that transfer to an identification process of the modal parameters. The bias error is located on the envelope of the modal correlation functions, thus violating the assumption that the correlation function matrix contains multiple free decays. Therefore, the bias error transmits to the damping estimates in operational modal analysis. In this paper, we show an automated algorithm that reduces the bias error caused by the statistical errors. This algorithm identifies erratic behaviour in the tail region of the modal correlation function and reduces this noise tail. The algorithm is tested on a simulation case and experimental data of the Heritage Court Building, Canada. Based on these studies, the algorithm reduces bias error and uncertainty on the damping estimates and increases stability in the identification process.
\end{abstract}

Keywords: Operational modal analysis, correlation function matrix, uncertainty, bias reduction, estimation error

\section{Introduction}

In operational modal analysis, we use the random vibrations of a linear and time-invariant system, excited by white Gaussian noise, to extract modal parameters. The correlation-driven operational modal analysis uses a two-stage time domain modal identification process [1]. The first step is the calculation of the correlation function matrix by the random vibrations of the system. In the second step, we treat the correlation function matrix as free decays of the system $[2,3]$ from which we estimate the modal parameters. Unfortunately, exact properties of any random data are inaccessible from sampled data with a finite length thus we must estimate them instead. In operational modal analysis, the finite length of the time series forces us to estimate the correlation function matrix and this introduces statistical errors [4]. The statistical errors are system dependent and they cause the estimated correlation function matrix to become a stochastic process that depends on the modal parameters and the time length. These statistical errors create random errors in the correlation functions [5-8] that increase with the number of time lags.

Recently, it was shown for operational modal analysis that the envelope of the correlation function is Rice distributed [5]. Therefore, the envelope becomes increasingly biassed as the random error increases in the estimated correlation function. This bias error results in erratic behaviour in the tail region of the estimated correlation function - referred to as the noise tail - for each mode in the system, see Fig. 1. The error depends on the frequency-damping product, the excitation level, and the time length of the measurement. Since the envelope is biassed in the noise tail, this introduces bias errors in the modal parameters from an

\footnotetext{
* Corresponding author

Email address: martar@eng.au.dk (Marius Tarp $\varnothing)$
} 
identification process. The bias error is pronounced for the estimated damping ratio since it is located at the envelope. The zero crossings of the correlation function is, however, unbiassed [5].

We should exclude the noise tails from an estimation of modal parameters to reduce the bias error in operational modal analysis. Each mode, however, has an individual position of the noise tail $[5,9,10]$ and this complicates the matter of choosing a sufficient length of the correlation function matrix when estimating the modal parameters. For instance, modes with a high frequency-damping product need a smaller part of the estimated correlation function than modes with a lower frequency-damping product to obtain a valid estimation [11]. Truncation, however, might lead to erroneous results if the correlation function has insufficient information about one of the modes for an estimation of the modal parameters [11].

In the literature, there are various methods for dealing with random noise on the system response. We can use singular value decomposition or principle component analysis for subspace reduction to remove noise perpendicular to the reduced subspace [12-14]. Research, however, indicates that a substantial part of the statistical error is located in the subspace of the physical signals $[5,10]$. Furthermore, we can remove the first part of the correlation function matrix to reduce the additive and uncorrelated noise [15]. The statistical errors in the correlation function matrix, however, are different than other types of random noise since it increases with time lags $[6,7]$. The use of exponential windows or other tapering windows, traditionally used to reduce leakage [16], could be applied to the correlation function matrix to reduce the noise tails [1]. Each mode, however, has a unique noise tail and this complicates the matter of choosing a suitable window (similar to a truncation of the correlation function matrix). Therefore, an exponential window might remove the noise tails from some modes while it tampers the physical response of others and loses information of these modes.

This article presents an algorithm for an automatic reduction of statistical errors in the estimated correlation function matrix while decreasing the chance of an erroneous truncation. This reduction of statistical errors assumes that we can manipulate the noise tail since its envelope is purely made of errors. We change the envelope in the noise tail to mimic that of the physical correlation function by using a least square fit of a negative exponential. The disadvantage of this algorithm is that the system must be overdetermined. So we need fewer modes than measured channels with the system response. We can, however, bypass this problem by applying filtering techniques to the response so the contribution of modes outside the filter is reduced. The algorithm reduces the bias on damping estimates and increases stability and reliability in the identification process of modal parameters.

\section{Theory}

\subsection{Correlation Function Matrix}

In operational modal analysis, we use ambient vibrations for modal analysis by measuring the spatial limited system response, $\mathbf{y}(t)$. We assume that stationary white Gaussian noise excites a linear and timeinvariant system [1]. When these assumptions are fulfilled, we can treat the correlation function matrix, $\mathbf{R}_{y}(\tau)$, as multiple free decays of the system $[2,3]$.

$$
\mathbf{R}_{y}(\tau)=\mathrm{E}\left[\mathbf{y}(t) \mathbf{y}^{\top}(t+\tau)\right]
$$

where E denotes the expectation operator.

Brincker [3] has calculated the analytic expression for the correlation, here written for positive time lags, $\tau$

$$
\mathbf{R}_{y+}(\tau)=2 \pi \sum_{i=1}^{N}\left(\boldsymbol{\gamma}_{i} \boldsymbol{\phi}_{i}^{\top} e^{\lambda_{i} \tau}+\boldsymbol{\gamma}_{i}^{*} \boldsymbol{\phi}_{i}^{H} e^{\lambda_{i}^{*} \tau}\right)
$$

where $\lambda_{i}$ is the modal pole, $\boldsymbol{\gamma}_{i}$ is the modal participation vector, and $\boldsymbol{\phi}_{i}$ is the mode shape for $i^{t h}$ mode.

If we apply the modal decomposition on the correlation function matrix and assume uncorrelated modal coordinates, we can decorrelate it into a diagonal matrix [1] where each diagonal component is a free decay of a single-degree-of-freedom (SDOF) system. The modal decomposition states that the response from a linear system is a linear combination of mode shapes and modal coordinates. 


$$
\mathbf{y}(t)=\mathbf{\Phi q}(t)
$$

where $\boldsymbol{\Phi}$ is the mode shape matrix and $\mathbf{q}(t)$ is a vector containing the modal coordinates for all modes. We insert Eq. (3) into Eq. (1).

$$
\mathbf{R}_{y}(\tau)=\boldsymbol{\Phi}_{q}(\tau) \boldsymbol{\Phi}^{\top}
$$

where $\mathbf{R}_{q}(\tau)$ is the modal correlation function matrix, which is based on the modal coordinates. In the case of uncorrelated modal coordinates, the modal correlation function matrix is a diagonal matrix where modal auto-correlation functions are equivalent to a free decay of a SDOF system of the given mode.

\subsection{Estimated Correlation Function Matrix}

When we have to calculate the correlation function matrix from measured data, we use time averaging instead of ensemble averaging since the system response is ergodic. The length of the signal must, however, tend towards infinity for this to be true [4].

$$
\mathbf{R}_{y}(\tau)=\lim _{T \rightarrow \infty} \frac{1}{T} \int_{0}^{T} \mathbf{y}(t) \mathbf{y}^{\top}(t+\tau) d t
$$

where $T$ is the total time length of the measured system response and ${ }^{\top}$ denotes the transpose.

In reality, however, we measure the system response with a sample time step, $\Delta t$, and a finite length of each data set. Therefore we have to estimate the discrete correlation function matrix from a finite sample size, which introduces statistical errors [4].

$$
\widetilde{\mathbf{R}}_{y}(k)=\frac{1}{N-k} \sum_{n=0}^{N-k} \mathbf{y}(n) \mathbf{y}^{\top}(n+k), \quad 0 \leq k<N
$$

where $\widetilde{\mathbf{R}}_{y}(k)$ is the estimated correlation function matrix, $N$ is the total number of samples, $T=N \Delta t, k$ is the discrete time lag, $\tau=k \Delta t$, and $\Delta t$ is the sampling interval.

This estimated correlation function matrix is a random variable with an expected value equal to the analytic correlation function matrix and a variance caused by the statistical errors which are additive Gaussian noise $[4,5]$. Since the finite time length of the recorded signal causes the statistical errors, the variance decreases when the time length increases [5-7]. Furthermore, the estimated correlation function matrix at different time lags is statistically dependent since we calculate the entire function matrix from the same sample. Thus, the statistical errors have cross-correlation.

For simplicity, let us assume that the statistical errors on the mode shapes are small and neglectable compared to the errors on the frequencies and damping ratios of the system. This enables us to decorrelate the statistical errors into individual statistical errors on the modal auto-correlation functions by the use of Eq. (4).

$$
\widetilde{R_{q_{i}}}(k)=\boldsymbol{\phi}_{i}^{\dagger} \widetilde{\mathbf{R}}_{y}(k)\left(\boldsymbol{\phi}_{i}^{\dagger}\right)^{\top}
$$

where ${ }^{\dagger}$ denotes the pseudo inverse and $\widetilde{R_{q}}(k)$ is the estimated modal correlation function.

Now, the variance on each modal auto-correlation function expresses the statistical errors that depend on the modal properties of the system, the excitation, and the duration of the system response [5, 7]. 

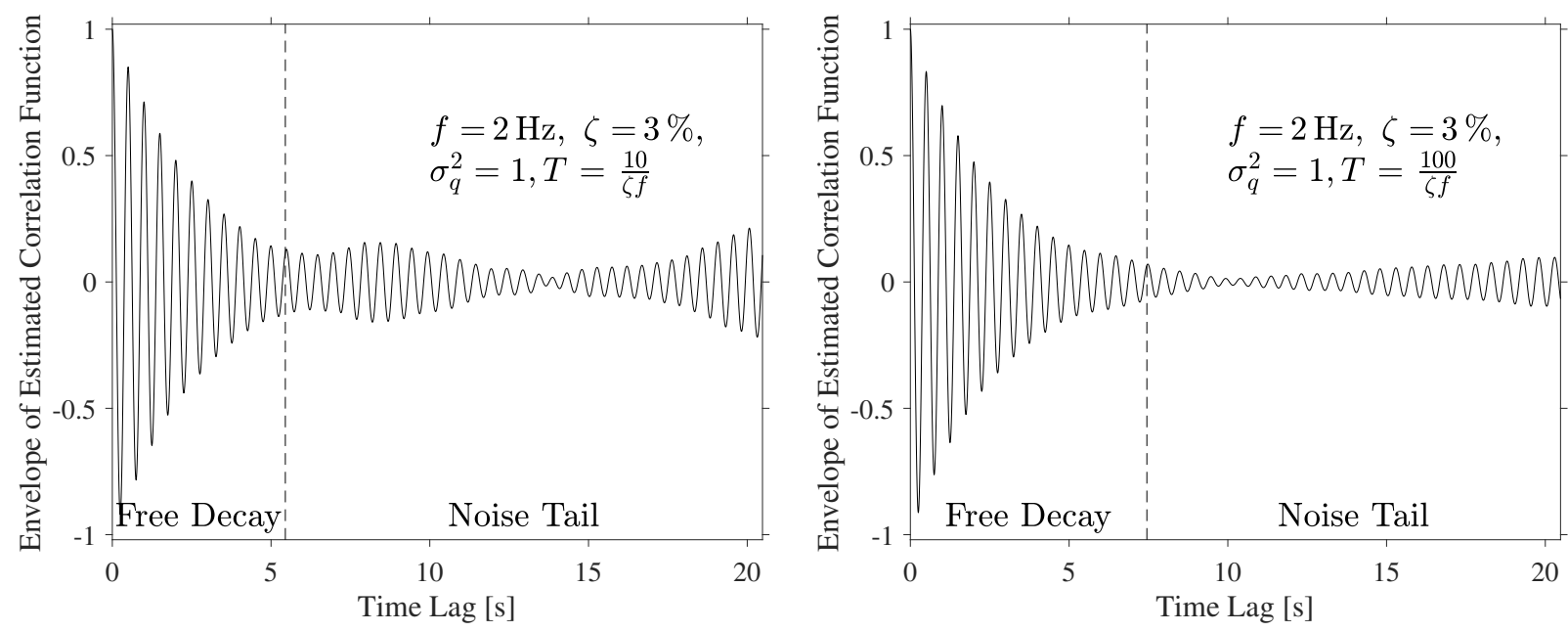

Figure 1: Correlogram with random iteration of the estimated correlation function for two different time lengths and the approximated beginning of the noise tail

\subsection{Noise Tail}

As previously mentioned, the statistical errors create erratic behaviour in the tail region of the estimated correlation function matrix - known as the noise tail - as illustrated in Fig. 1. This happens when the statistical errors dominate the correlation function $[5,7,9,10]$. Tarpø et al. [5] showed that the envelope of the estimated modal correlation function is Rice distributed and therefore biassed in the tail region, see Fig. 2. The discrete expected value of the envelope follows this expression

$$
\operatorname{env}_{i}(k)=\sqrt{\frac{\pi}{2}} \sigma_{q_{i}}^{2} \sqrt{\frac{1+e^{-2 \omega_{i} \zeta_{i} k \Delta t}\left(2 \omega_{i} \zeta_{i} k \Delta t+1\right)}{2(N-k) \Delta t \omega_{i} \zeta_{i}}} L_{1 / 2}\left(\frac{(k-N) \Delta t \omega_{i} \zeta_{i}}{e^{2 \omega_{i} \zeta_{i} k \Delta t}+2 \omega_{i} \zeta_{i} k \Delta t+1}\right)
$$

where $L_{1 / 2}(\cdot)$ is the Laguerre polynomial for case $\frac{1}{2}, \sigma_{q_{i}}^{2}$ is the variance of the modal coordinates, $\omega_{i}$ is the natural frequency, and $\zeta_{i}$ is the damping ratio for the $i^{\text {th }}$ mode. Fig. 2 illustrates the expected envelope of the estimated correlation function, the envelope of the analytic correlation function, and the bias error caused by the statistical errors [5].

Fig. 3 shows a random realisation of an estimated correlation function for two different time lengths. The envelope of an analytic free decay is plotted along with the expected value of the envelope from Eq. (8). The frequency of the oscillations from the correlation functions is consistent, at least to the eye, in Fig. 3 whereas the envelope is obviously biassed in the tail region. Therefore, the envelope of an estimated correlation function indicates the level of statistical errors since it diverts from a negative exponential function as the errors increase.

The expected envelope has an increasing bias error as the time lag of the correlation function increases. When the statistical errors are small, the expected envelope is an approximated exponential decay. As the statistical errors start to dominate, the envelope becomes constant and this creates the noise tail thus violating the assumption that the correlation function matrix consists of free decays. Therefore, it is essential that we reduce the statistical errors in operational modal analysis.

Since statistical errors affect each mode in the correlation function, it creates a noise tail for each mode in the estimated correlation function matrix. As seen in Eq. (8), the expected value of the modal envelope will be different for each mode. Therefore, the individual noise tails start at different positions for each mode. This poses a problem when we have to choose the number of time lags to utilise from the correlation function matrix. If we use a small part of the correlation function matrix to remove noise tail for one mode, it might truncate another mode and cause an erroneous identification of that given mode. This problem is further discussed in [11]. 

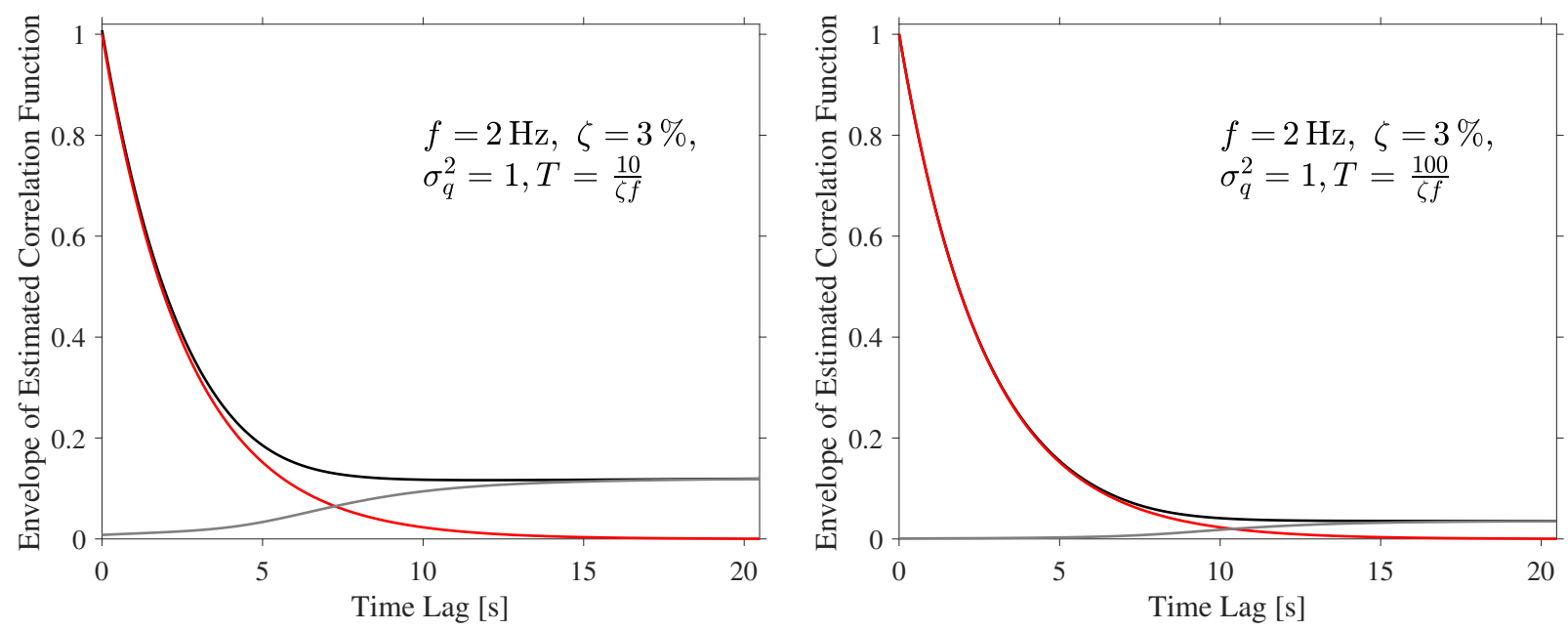

Figure 2: The expected value of the envelope of the estimated correlation function (black), the envelope of the correlation function, Eq. (2), (black), and the difference between them (the bias error) (grey) for two different time lengths
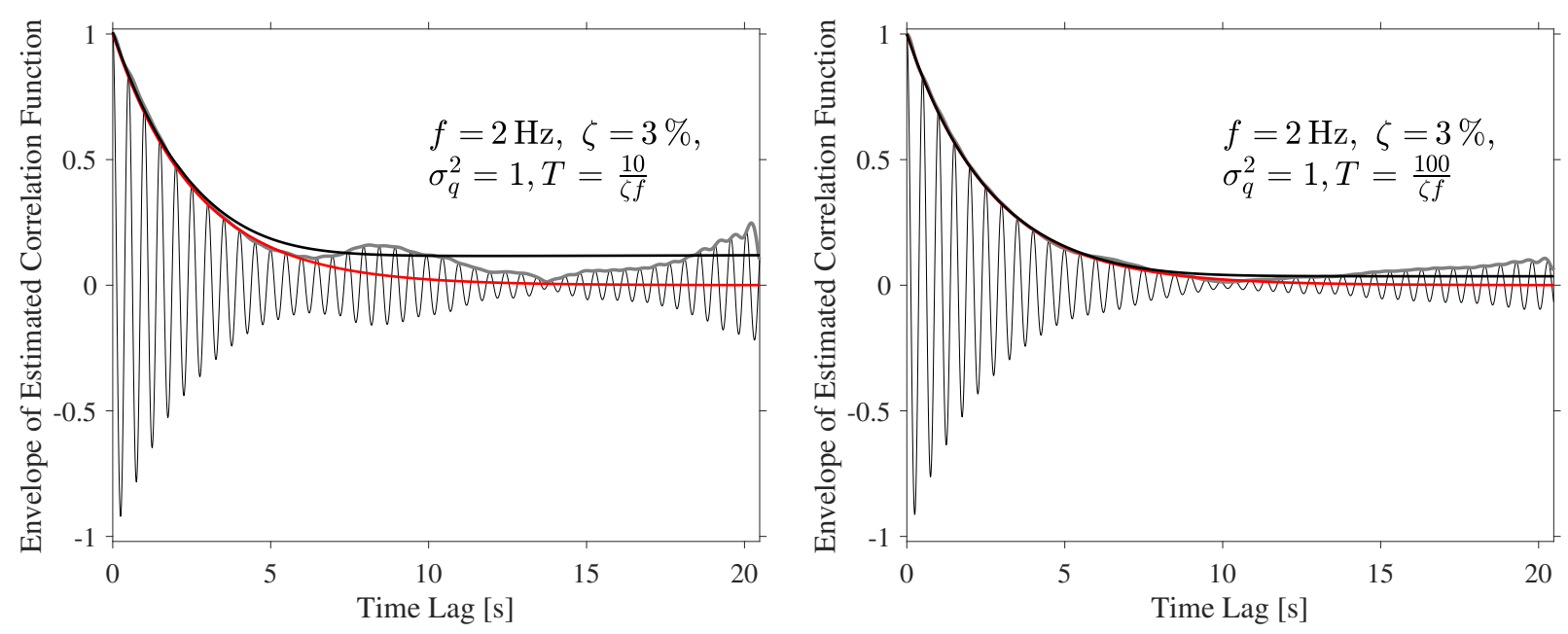

Figure 3: Correlogram with random iteration of the estimated correlation function with the expected value of the envelope (black), the estimated envelope (grey), and the envelope of the correlation function, Eq. (2), (black) for two different time lengths 


\section{Algorithm}

In this section, we will introduce a novel algorithm to minimise the bias error caused by the statistical errors without truncating the correlation function matrix. The algorithm decorrelates the correlation function matrix, detects the noise tails, and modifies the envelopes of the decorrelated correlation functions in these regions.

\subsection{Modal Decorrelation of Correlation Function Matrix}

We want to decorrelate the estimated correlation function matrix into an approximated modal correlation function matrix as in Eq. (7). This decorrelation is called a similarity transformation. When the transformation matrix contains the eigenvectors as column vectors, the similarity transformation diagonalise the estimated correlation function matrix.

We could use any identification process to identify mode shapes and apply these to decorrelate and equalise the energy in the estimated correlation function matrix. Thus, the algorithm is applicable on previously identified modal parameters to reduce uncertainty of these since we can apply the identified mode shapes in the modal decorrelation of the estimated correlation function matrix. In this article, we automatically estimate the mode shapes using a condensation technique by Olsen et al. [12] combined with a similar identification process as Vold et al. [17] using auto-regression models and a poly reference technique, see Sec. Appendix A.

When we have a transformation matrix, $\tilde{\boldsymbol{\Phi}}$, containing a set of identified mode shape, we decorrelate the estimated correlation function matrix into an approximated modal correlation function matrix, which we will call the decorrelated correlation function matrix.

$$
\widehat{\mathbf{R}}_{q}(\tau)=\tilde{\boldsymbol{\Phi}}^{\dagger} \widetilde{\mathbf{R}}_{y}(\tau)\left(\tilde{\boldsymbol{\Phi}}^{\dagger}\right)^{\top}
$$

On the diagonals of this matrix, the correlation functions are decorrelated to auto-correlation functions, which corresponds to free decays of SDOF systems. Since this is an approximated decorrelation, the decorrelated correlation function matrix is a non-diagonal matrix.

\subsection{Envelope Detection}

In Signal Processing we can use the Hilbert transformation as envelope detection [18]. For this algorithm, we want to find the envelopes of the diagonal components of the decorrelated correlation function, Eq. (9). These diagonal components, $\widehat{R_{q_{i}}}(k)$, are auto-correlation functions and they each form an approximated free decay of a SDOF system.

When we apply the Hilbert transformation, we obtain a phase shifted signal, $\mathcal{H}\left[{\widehat{R_{q}}}_{q}(k)\right]$. We estimate the envelope of each decorrelated auto-correlation functions using the absolute value of the analytic signal $[18]$.

$$
e_{i}(k)=\sqrt{\widehat{R}_{q_{i}}(k)^{2}+\mathcal{H}\left[\widehat{R}_{q_{i}}(k)\right]^{2}}
$$

where $\mathcal{H}$ denotes the Hilbert transformation. Fig. 3 shows two examples of the Hilbert envelope of a decorrelated correlation function. The decorrelated auto-correlation functions are aperiodic functions since they have a discontinuity in the ends due to the noise tail. Therefore, oscillations often exist in the Hilbert envelope at the end of the correlation functions, see Fig. 3. These (Gibbs) oscillations must be positioned in the noise tail since they scramble the detection of the real noise tail. 


\subsection{Identifying the location of the Noise Tail}

The envelope of the estimated correlation function is a stochastic process that follows a Rice distribution [5]. The analytic expression for the expected envelope is a Laguerre polynomial, Eq. (8), and each realization of the stochastic process varies slightly. Generally, the physical part of a modal correlation function resembles an exponential decay while the noise tail has an almost constant envelope with fluctuations. Thus, the logarithmic envelope of the physical part is similar to a straight declining line and the noise tail is the divergence that produces a near constant envelope. This, however, is an approximation since the shift from the physical part to the noise tail is gradual due to the Laguerre polynomial.

We use the updated algorithm of Tarpø et al. [9] to find the position of the noise tail. It fits two lines to the estimated envelope where the transition between the two lines is a variable. The combined model has four parameters for each transition between the two lines, and the best fit of all the possible transitions indicates the beginning of the noise tail. Since the expected envelope is described by a Laguerre polynomial, this combined model is a simplification that locates the noise tail as the statistical error dominates completely.

Firstly, we divide the estimated envelope for a decorrelated auto-correlation function into two vectors for each line as a function of the transition, $n$.

$$
\begin{aligned}
& \mathbf{m}_{1}(n)=\log \left(\left[\begin{array}{llll}
e_{i}(1) & e_{i}(2) & \ldots & e_{i}(n)
\end{array}\right]\right)^{\top} \\
& \mathbf{m}_{2}(n)=\log \left(\left[\begin{array}{llll}
e_{i}(n+1) & e_{i}(n+2) & \ldots & e_{i}(N)
\end{array}\right]\right)^{\top}
\end{aligned}
$$

We use linear regression to fit the two lines to the logarithmic envelope.

$$
\mathbf{m}_{i}(n)=\mathbf{X}_{i}(n) \mathbf{a}_{i}(n)
$$

We set up design matrices, $\mathbf{X}_{i}(n)$, between the two lines.

$$
\begin{gathered}
\mathbf{X}_{1}(n)=\left[\begin{array}{ccccc}
1 & 2 & 3 & \ldots & n \\
1 & 1 & 1 & \ldots & 1
\end{array}\right]^{\top} \\
\mathbf{X}_{2}(n)=\left[\begin{array}{ccccc}
n+1 & n+2 & n+3 & \ldots & N \\
1 & 1 & 1 & \ldots & 1
\end{array}\right]^{\top}
\end{gathered}
$$

We find the regression parameters by Least Squares [19]:

$$
\hat{\mathbf{a}}_{i}(n)=\mathbf{X}_{i}^{\dagger}(n) \mathbf{m}_{i}(n)
$$

So we have the best fit for both lines.

$$
\hat{\mathbf{m}}_{i}(n)=\mathbf{X}_{i}(n) \hat{\mathbf{a}}_{i}(n)
$$

We compile the two estimated lines into one vector.

$$
\hat{\mathbf{e}}(n)=\exp \left(\left[\begin{array}{c}
\hat{\mathbf{m}}_{\mathbf{1}}(n) \\
\hat{\mathbf{m}}_{\mathbf{2}}(n)
\end{array}\right]\right)
$$

We use the Coefficient of Determination [20] for each iteration of $n$ as a quality quantification.

$$
r^{2}(n)=1-\frac{\sum_{k=1}^{N}\left(e_{i}(k)-\hat{e}_{i}(k)\right)^{2}}{\sum_{k=1}^{N}\left(e_{i}(k)-\mathrm{E}[\mathbf{e}]\right)^{2}}
$$

We repeat this process for all possible values of $n$. The highest value of the Coefficient of Determination indicates the best fit and thereby the start of the noise tail, $n_{0}$.

$$
r^{2}\left(n_{0}\right)=\max \left(r^{2}(1), r^{2}(2), r^{2}(3), \ldots, r^{2}(N)\right)
$$



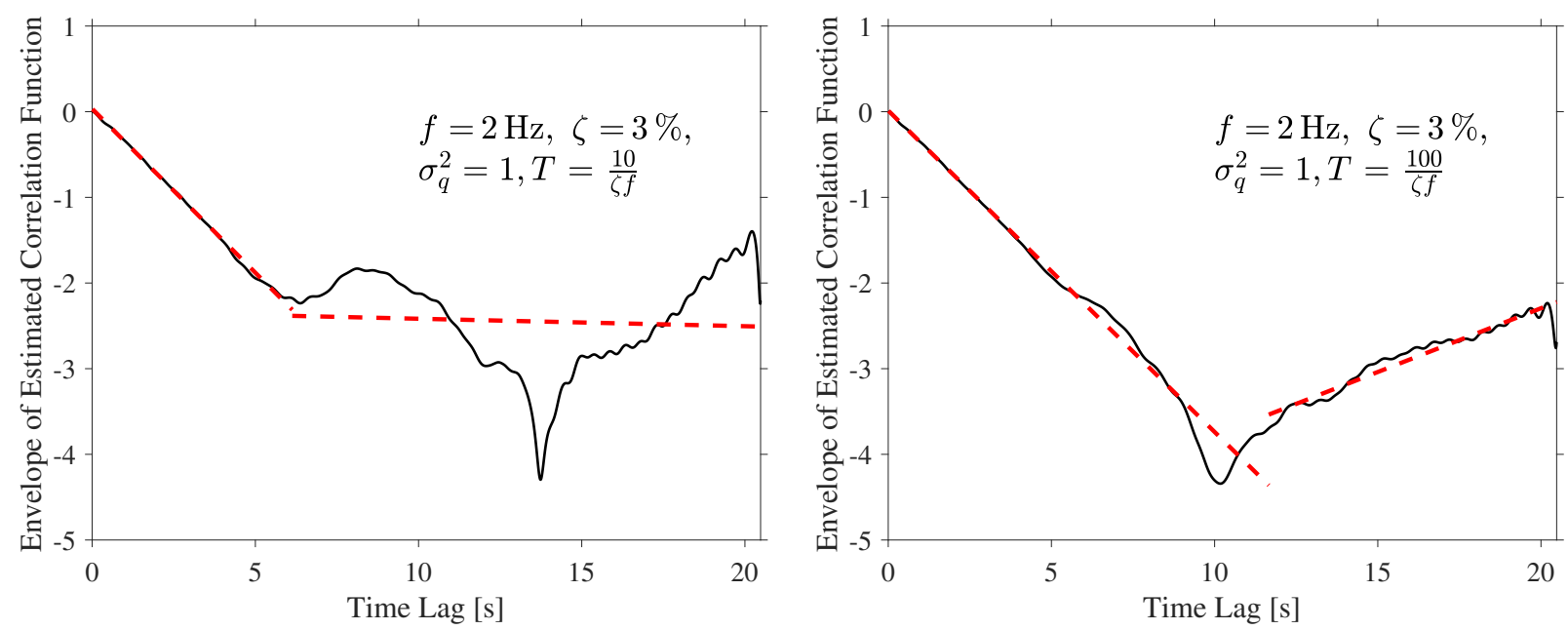

Figure 4: Best fit on the analytic mean value of the envelope, the estimated Hilbert envelope of the correlation functions from Fig. 3 (black line) and the two fitted models (red) for two different time lengths

Fig. 4 illustrates the best fit of these two models on two different estimated correlation functions based on system responses with different time lengths. Although the piecewise linear fit seems insufficient as a fit of the logarithmic envelope (especially in the noise tail region), it approximates the point of transition into the noise tail well.

Since we estimate the location of the noise tail where the statistical errors dominate, then we have substantial bias error in the part right before the estimated noise tail. In order to reduce this bias error, we say the physical part is located before the noise tail with a transition area between the two. The authors obtained good results with the following expression for the end of the physical part: $n_{1}=0.8 n_{0}$.

\subsection{Modification of the Noise Tail}

We use the envelope of the identified physical part of the correlation functions to modify the envelope of the noise tail. The regression gave the parameters of the line corresponding to the best fit of the physical envelope and we extend this regression line to the envelope of the noise tail by modifying the original envelope. We have two parameters for the line in the physical part, $\hat{\mathbf{a}}_{1}\left(n_{1}\right)$. We extend the best fit of the free decay into the noise tail. Thereby, we obtain a modified envelope where we only change the envelope of the noise tail based on the best fit of the physical part of the envelope.

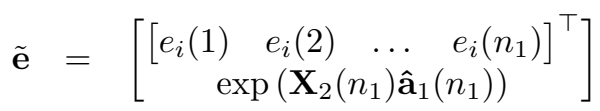

The transition between the two combined envelopes often has abrupt changes that need to be smoothen since they disrupt the identification process of the modal parameters. We define a transition region, $n_{1} \pm \frac{n_{1}}{6}$, and we apply a monotone piecewise cubic interpolation [21] to replace this region by an interpolation using the rest of the modified envelope, ẽ.

Fig. 5 shows two examples of a modified envelope for a decorrelated correlation function based on different time lengths. The modified envelope is identical to the original envelope for discrete time lags below $n_{1}$.

We substitute this modified envelope with the original envelope for each diagonal component in the decorrelated correlation function matrix.

$$
{\stackrel{*}{R_{q_{i}}}}_{(k)}={\widehat{R_{q_{i}}}}_{(k)} \frac{\tilde{\mathbf{e}}(k)}{\mathbf{e}(k)}
$$



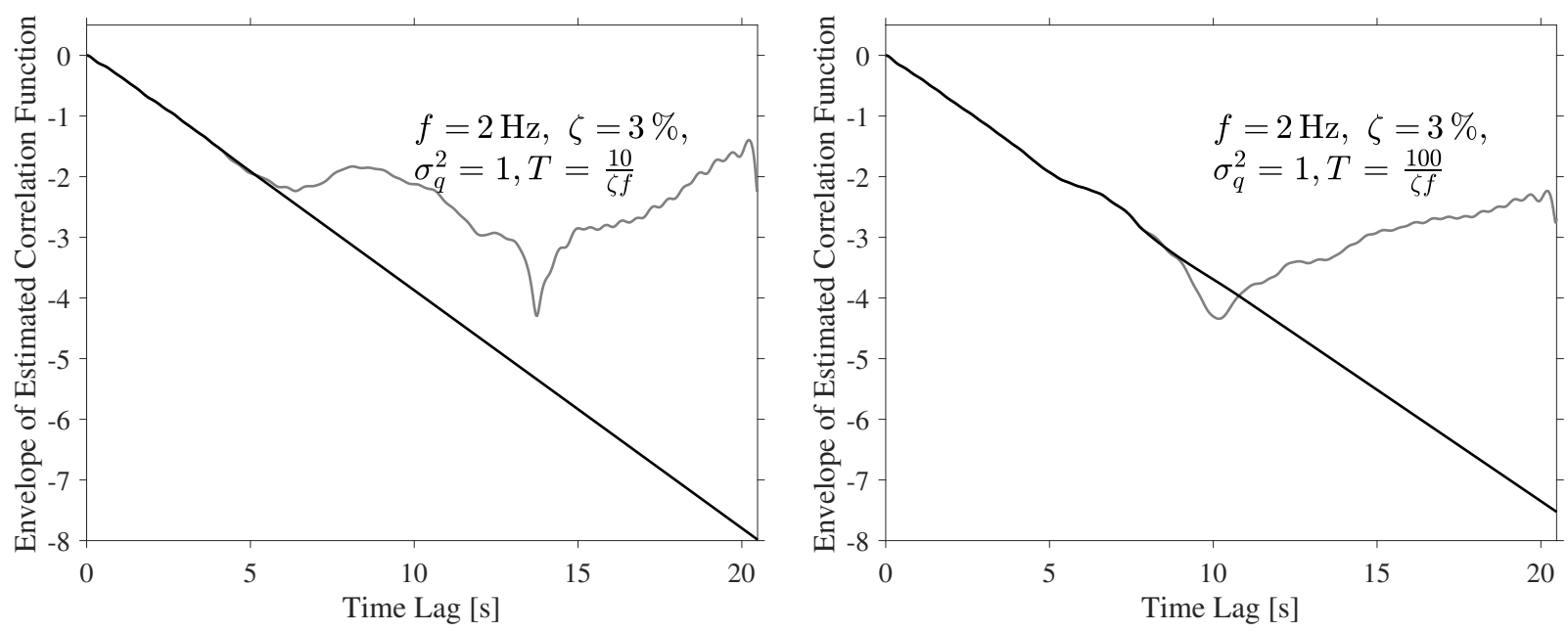

Figure 5: Modified Hilbert envelope (black) and the estimated Hilbert envelope (grey) of the correlation functions from Fig. 3 in logarithmic scale for two different time lengths
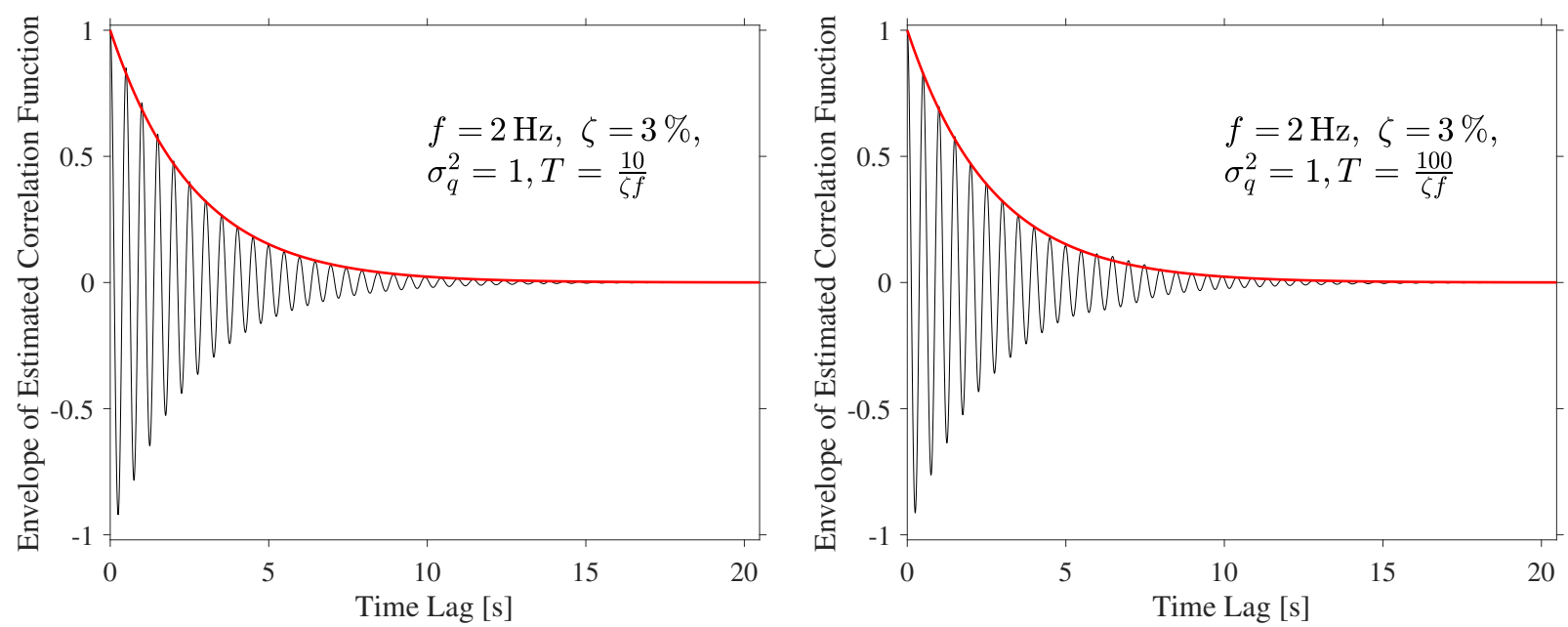

Figure 6: Correlogram with the modified estimated correlation function (black) based on the correlation functions from Fig. 3 with the analytic envelope of a free decay corresponding to the modal parameters (black) for two different time lengths 


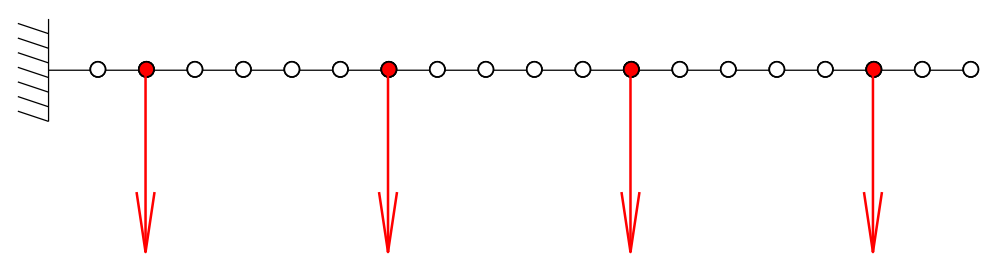

Figure 7: Case 1 - Simulated system: Illustration of the cantilever beam used in the simulation study and the position of the sensors (black)

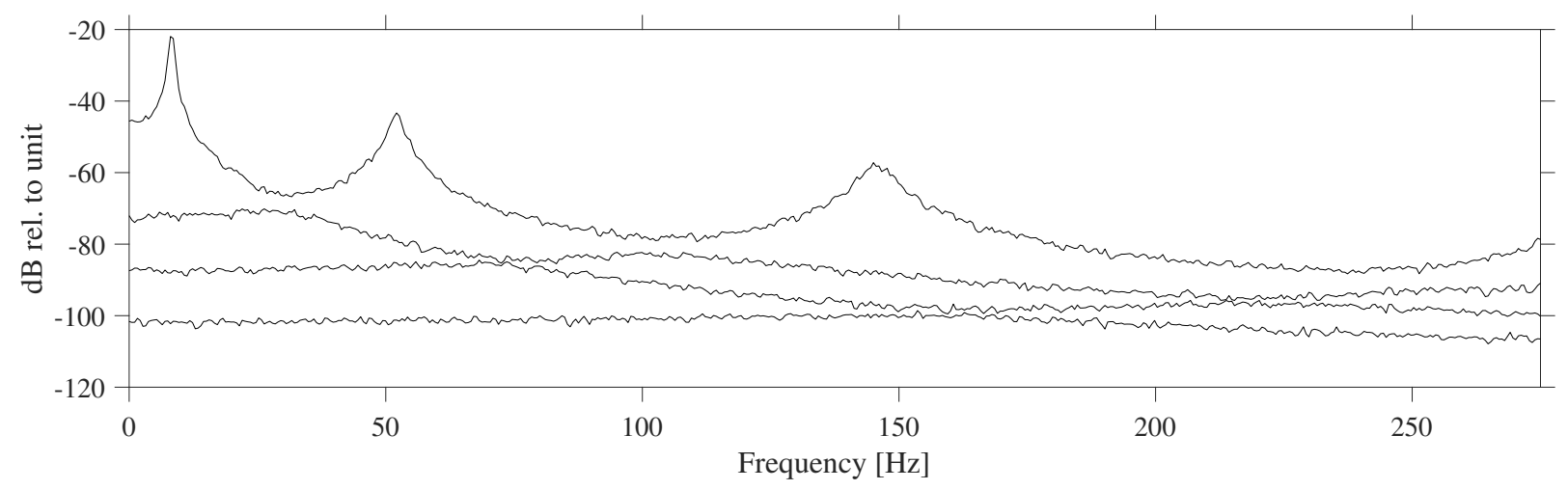

Figure 8: Case 1 - Simulated system: Singular values of the spectral density matrix of a random iteration of the Monte Carlo simulation using the Welch technique with $50 \%$ overlap

Finally, we truncate $\stackrel{*}{\mathbf{R}}_{q}(k)$ so it excludes time lags higher than the detected noise tails. In other words, the noise tail located at the highest time lag determines the truncation. Then we can use this modified correlation function matrix in an identification process. Fig. 6 illustrates the modification of a correlation function.

\section{Case Studies}

\subsection{Case 1 - Simulated Cantilever Beam}

We simulate the response of a cantilever beam in 2D, made with 20 Euler-Bernoulli Beam elements, using the Fourier transformed superposition method with all modes implemented in the OMA-toolbox from [1]. To mimic the spatial limited response measured by sensors, we extract the responses of four translational degrees-of-freedom, corresponding to four sensors, see Fig. 7. Since we are interested in the first three modes of the system, see Tab. 1, we use a sampling frequency of $550 \mathrm{~Hz}$ and a time length of 60 seconds, using the recommended time length from [1]. We run 10,000 simulations of the system response excited by white Gaussian noise in all degrees-of-freedom and we calculate the correlation function matrix for each iteration using Eq. (6). The singular values of the spectral density matrix are plotted in Fig. 8 for a random simulation.

To estimate the modal parameters from an estimated correlation function matrix, we use the Time Domain Poly-reference [17], the Ibrahim Time Domain [22], and the Eigensystem Realization Algorithm [13] from the OMA-toolbox in [1] with two different model orders. For each simulation, we estimate the modal parameters from the original correlation function matrix. We apply the algorithm to reduce the statistical errors of the correlation function matrix automatically and we estimate a new set of modal parameters. Then we choose the estimated modes with modal parameters closest to the three real modes. 
The number of included time lags of the correlation function matrix is important for the estimation of modal parameters since the statistical errors increase with increasing number of time lags. These errors create increasing random errors on the estimated correlation functions and increasing bias errors on the estimated envelope [5]. Therefore, the number of time lags is important in an analysis of this algorithm. This complicates the comparison of the normal identification procedure and identification based on this algorithm. So, we test different lengths of the correlation function matrix but we choose 513 discrete time lags $(1.03 \mathrm{~s})$ to illustrate the algorithm in the figures.

Table 1: Case 1 - Simulated system: Modal parameters of the system

\begin{tabular}{cccc}
\hline & Mode 1 & Mode 2 & Mode 3 \\
\hline Frequency $[\mathrm{Hz}]$ & 8.32 & 52.1 & 145.9 \\
Damping Ratio [\%] & 2 & 2 & 2 \\
\hline
\end{tabular}

\subsubsection{Results and discussion}

Figs. 9 and 10 display the estimated modal parameters from the original and the modified correlation function matrix for two different model orders. For the original correlation function matrices, large bias errors and skewness exist in the distribution of modal parameters for all identification techniques with a model order of two. By increasing the model order to three, the estimates of the third mode become approximately Gaussian distributed and the bias error decreases. For the modified correlation function matrix, the distribution is consistently approximately Gaussian and the bias error of the damping estimates is lower regardless of the model order.

The different identification techniques have diverse bias and random errors for the third mode, indicating that the noise tail influences the techniques differently when the model order is low. By increasing the model order, we decrease the skewness of the estimates. So an identification technique with a higher model order is better able to fit with a weakly-excited mode with a large noise tail. All the techniques, however, provide similar results with a low model order when we use the algorithm to reduce the noise tail. Furthermore, an identification technique with low model order has lower bias error using the modified correlation function matrix than a technique with a slightly higher model order using the original correlation function. So the algorithm creates stability in terms of the model order in the identification process.

For the first and second mode, the effect of the algorithm is modest with the respective number of time lags of 513. The uncertainty or skewness of the estimation is mostly indifferent to the algorithm. The second mode, however, gets a sample mean of the frequency and damping estimates closer to the real value for the low model order. For the high model order, the algorithm affects the bias on the damping estimates for the second mode. This indicates a general reduction of bias error, regardless of the identification technique and the model order.

In Figs. 9 and 10, the algorithm has an influence on the estimation of the modal parameters for the third mode. The bias errors reduce when the model order is low. Here a substantial change appears in the distribution of the modal parameters for the third mode. Both damping and frequency estimates have a skewed distribution for the original correlation function matrix, whereas the skewness ceases for the modified correlation function matrix, see Fig. 9.

Figs. 11 and 12 illustrate the results of an identification process with varying number of included time lags. Regardless of the model order, the bias and random errors rise with the increasing inclusion of the noise tails - this is especially apparent for the second and third mode. Evidently, the low model order has increasing estimation errors on the third mode and the Time Domain Poly-reference technique overestimates the damping ratio and underestimates the natural frequency, see Fig. 11. For a model order of three, the identification technique underestimates the damping ratio and natural frequency, see Fig. 12. Furthermore, for a small number of included time lags, the identification procedure slightly overestimates the damping ratio for the first mode, indicating truncation errors. The algorithm creates considerably more stable estimates than the original correlation function matrix regardless of the number of included time lags, see Fig. 11. 

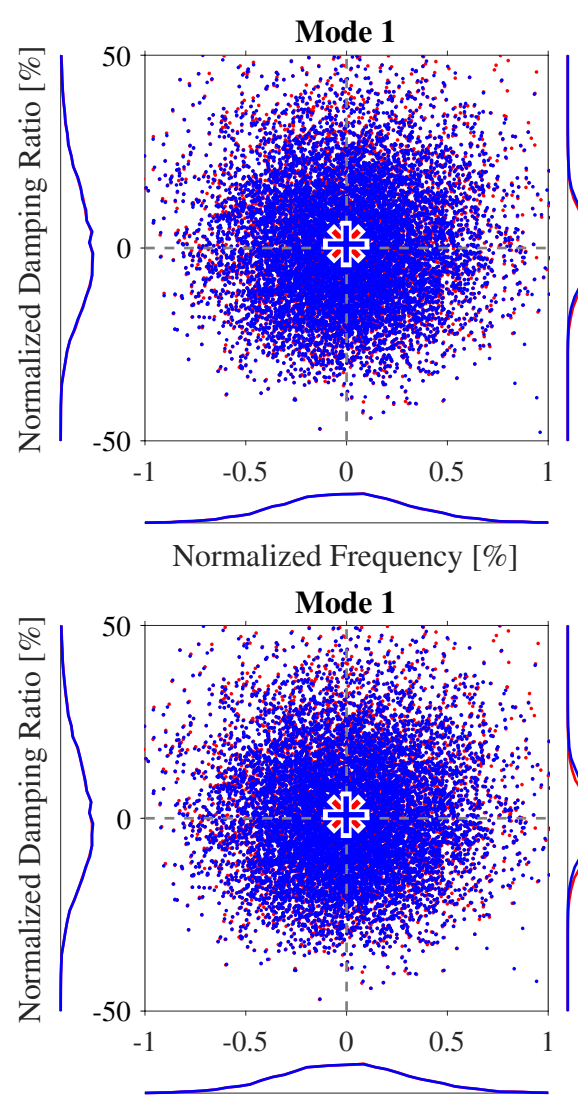

Normalized Frequency [\%]

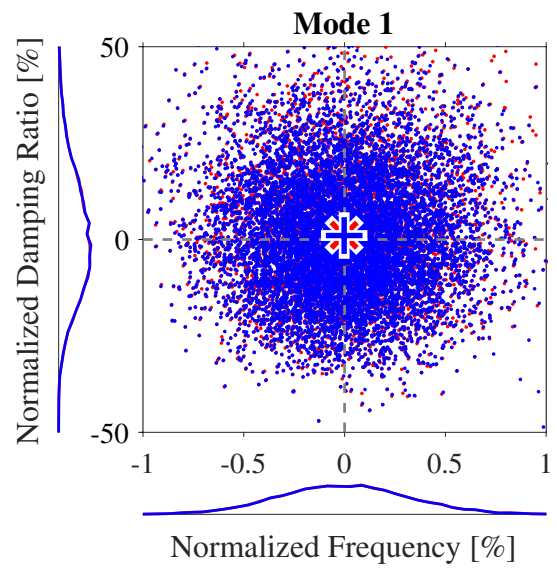

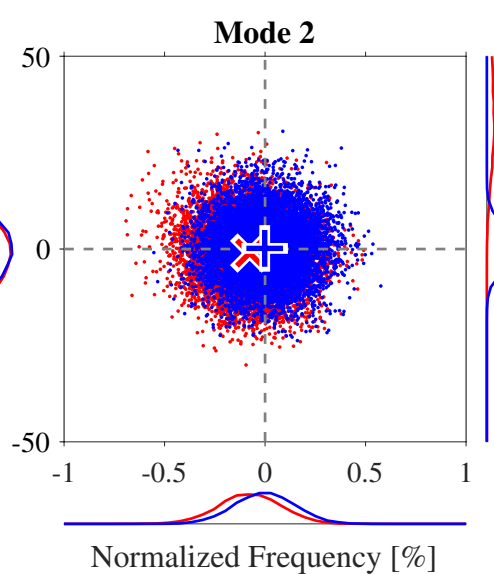

Mode 2

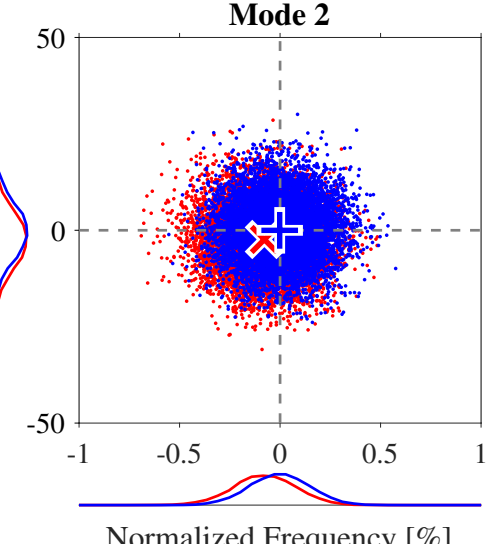

Normalized Frequency [\%]

Mode 2

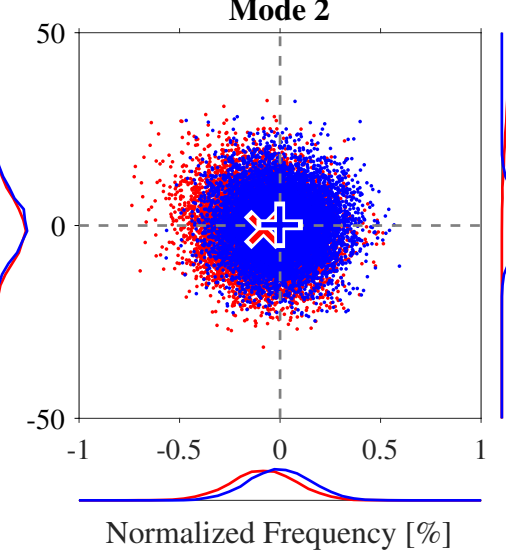

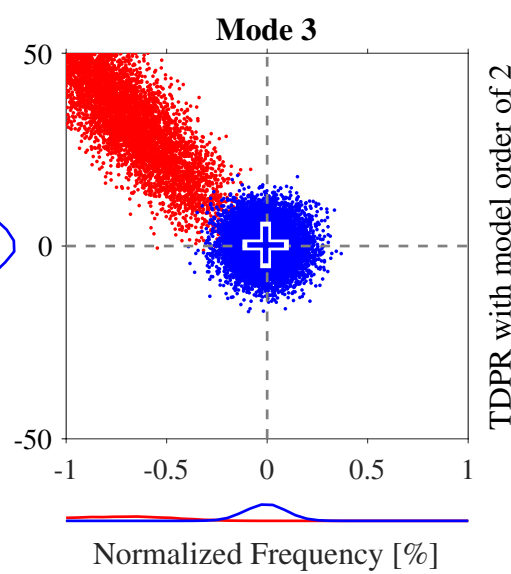

Mode 3

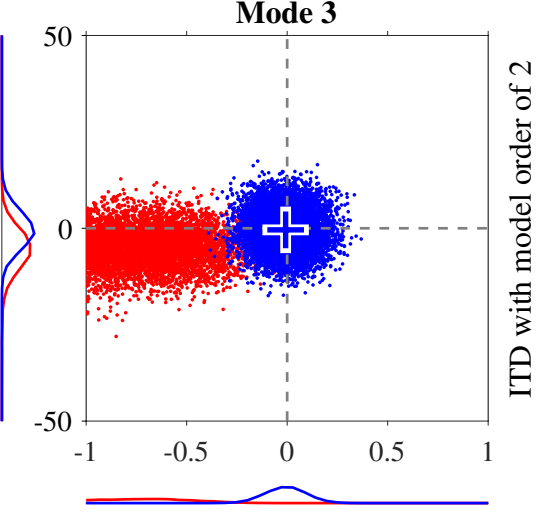

Normalized Frequency [\%]

Mode 3

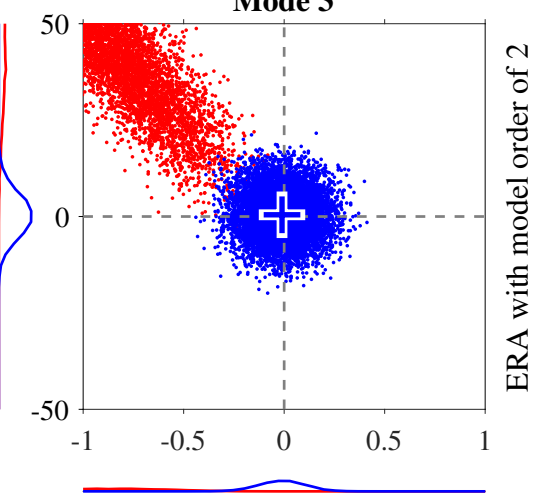

Normalized Frequency [\%]

Figure 9: Case 1 - Simulated system with 10,000 simulations: Identification of modal parameters from the first 513 time lags from the original correlation function matrix (black) with sample mean (black "x"), and the modified correlation function matrix (blue) with sample mean (blue "+") using Time Domain Poly-reference (TDPR), Ibrahim Time Domain (ITD), and the Eigensystem Realization Algorithm (ERA) for a model order of 2 

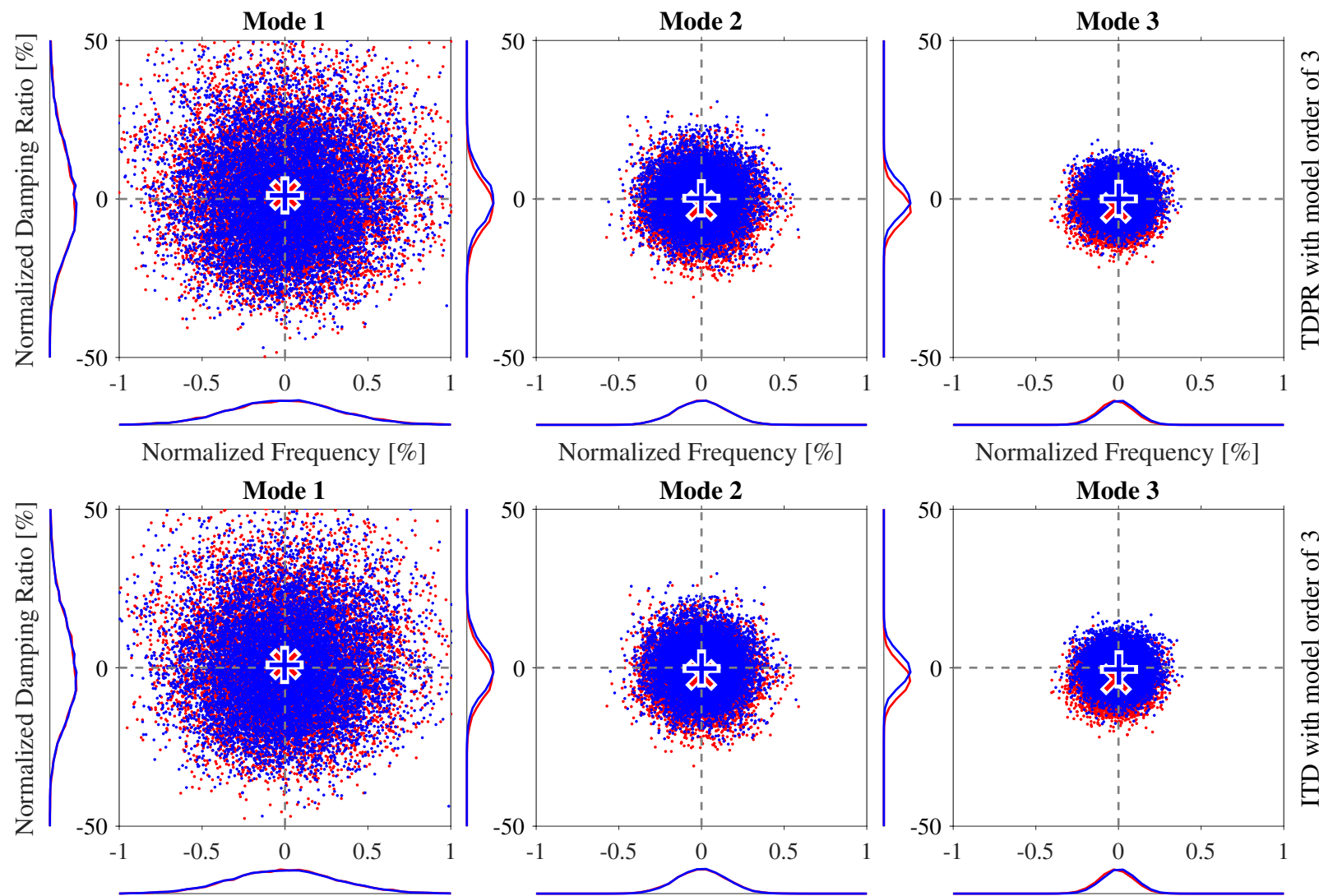

Normalized Frequency [\%]

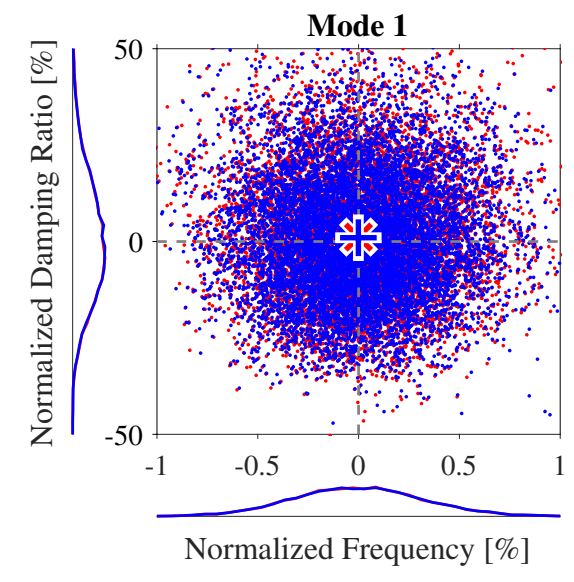

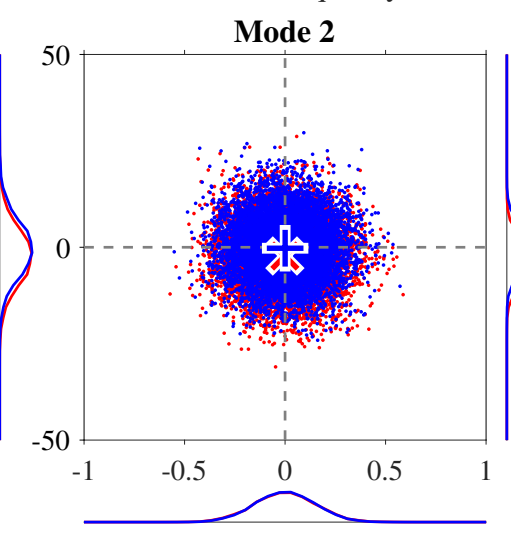

Normalized Frequency [\%]

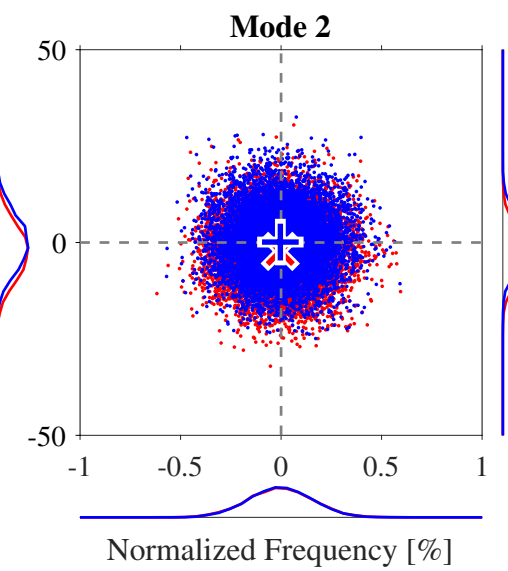

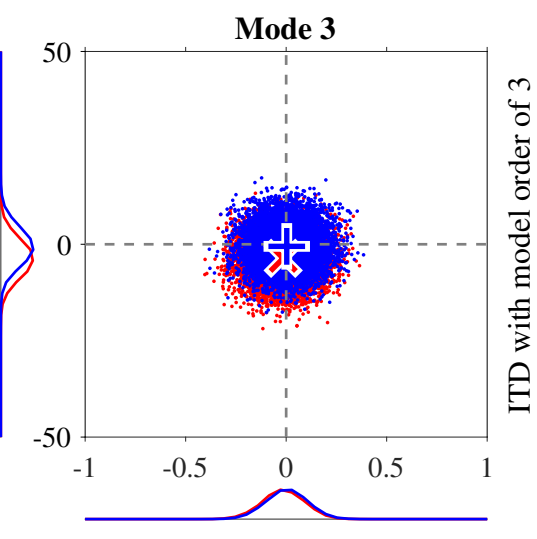

Normalized Frequency [\%] Mode 3

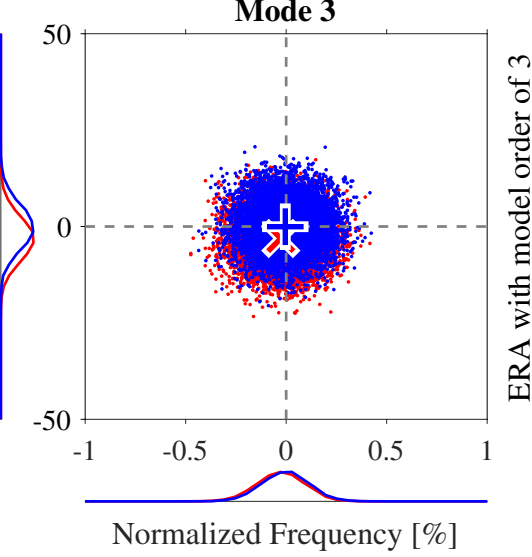

Figure 10: Case 1 - Simulated system with 10,000 simulations: Identification of modal parameters from the first 513 time lags from the original correlation function matrix (black) with sample mean (black "x"), and the modified correlation function matrix (blue) with sample mean (blue "+") using Time Domain Poly-reference (TDPR), Ibrahim Time Domain (ITD), and the Eigensystem Realization Algorithm (ERA) for a model order of 3 

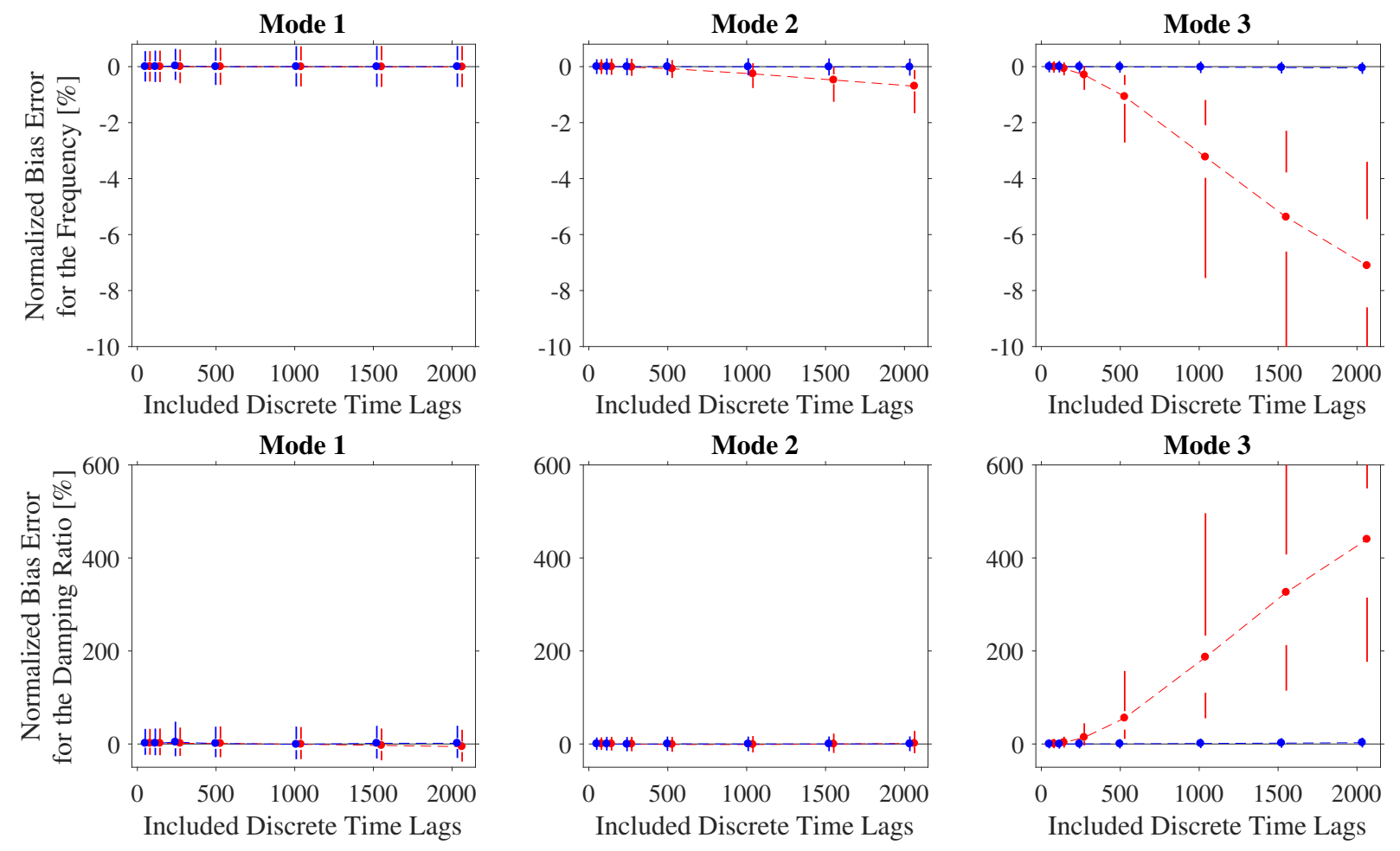

Figure 11: Case 1 - Simulated system with 10,000 simulations: (Tufte) box-plot of the estimated modal parameters using Time Domain Poly-reference a with model order of two [17] for a varying number of included time lags. Estimates based on the original (black) and the modified correlation function matrix (blue). In the (Tufte) box-plot, the dots mark sample means and the vertical lines mark the $2^{\text {nd }}, 25^{\text {th }}, 75^{\text {th }}$, and $98^{\text {th }}$ percentiles.

The algorithm has a substantial influence on the bias error in the estimation of modal parameters. Furthermore, it creates a more stable identification process since it, in this study, seems insensitive to the number of included time lags and the model order of the identification technique.

\subsection{Case 2 - Heritage Court Building}

We will look at the algorithm applied to a modal analysis of the Heritage Court Tower, dataset 1 [1]. The Heritage Court Building is a 15-story building with a reinforced concrete shear core in Vancouver, British Columbia, Canada, see Fig. 13. On April $28^{\text {th }}$ 1998, operational modal tests were carried out to analyse the dynamic properties of the building. A total of eight accelerometers were used in the test where six sensors were rowed while the other two sensors were used as references located on the $14^{\text {th }}$ floor. The vibration measurements have a sampling frequency of $50 \mathrm{~Hz}$ and a time length of 326 seconds. This is a relatively short time length so the level of statistical errors will be significant.

For this study, we will focus on the first dataset with a total of six sensors. A band-pass filter is used with cut-off frequencies of 0.95 and $1.75 \mathrm{~Hz}$ and roll-off bands of $0.4 \mathrm{~Hz}$.

We use the Eigensystem Realization Algorithm [13] on the correlation function matrix to create a stability diagram by changing the model order (the number of block rows in the Hankel matrices) while we keep the number of extracted modes the same as the number of channels in the measurements. We compare the stability diagram based on the original correlation function matrix with the stability diagram from the modified correlation function matrix where the algorithm reduces the noise tails automatically. For both stability diagrams, the correlation function matrix has 500 discrete time lags and we omit the first ten discrete time lags to remove uncorrelated broadband noise [15]. 


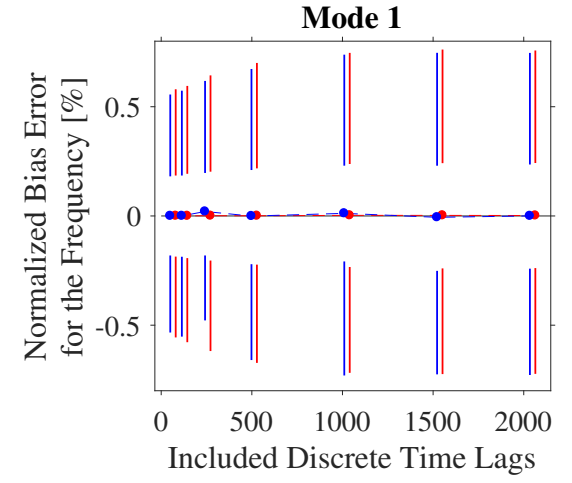

Mode 1

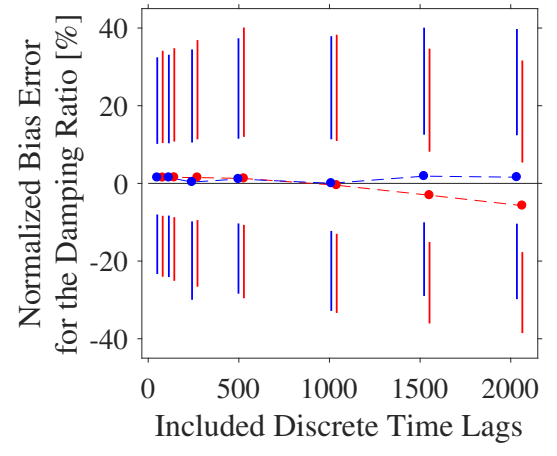

Mode 2

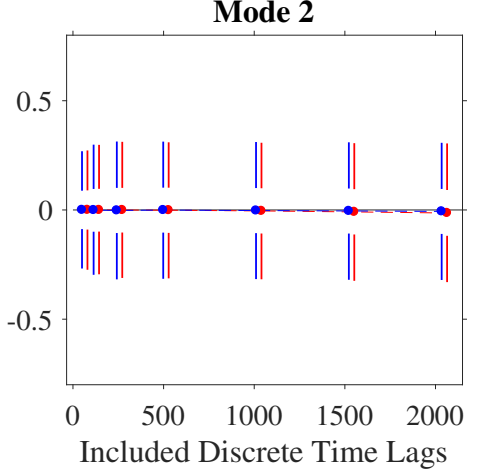

Mode 2

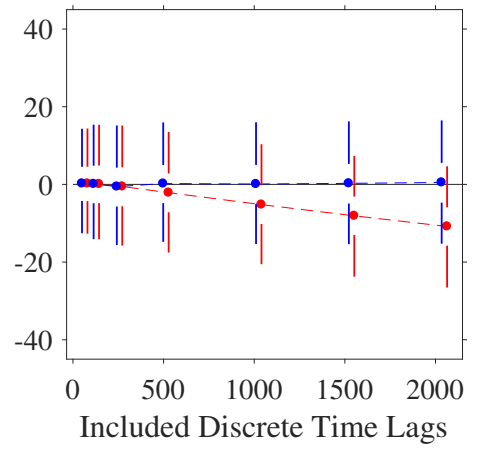

Mode 3

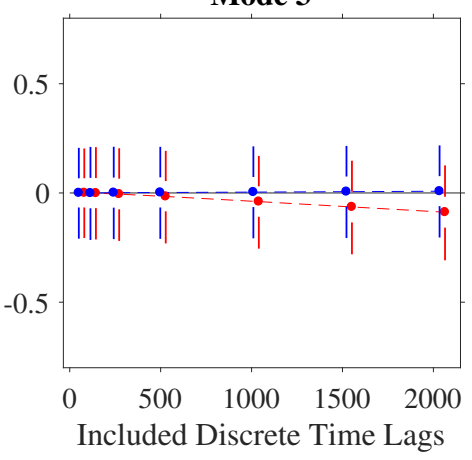

Mode 3

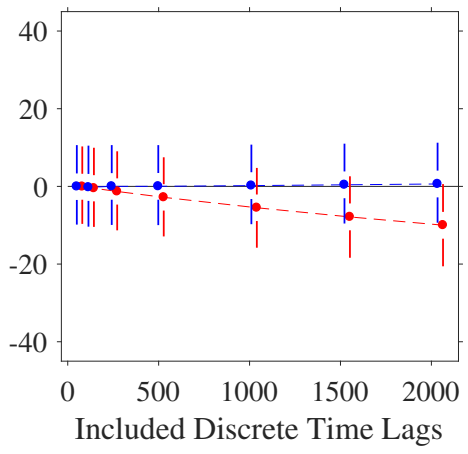

Figure 12: Case 1 - Simulated system with 10,000 simulations: (Tufte) box-plot of the estimated modal parameters using Time Domain Poly-reference with a model order of three [17] for a varying number of included time lags. Estimates based on the original (black) and the modified correlation function matrix (blue). In the (Tufte) box-plot, the dots mark sample means and the vertical lines mark the $2^{\text {nd }}, 25^{t h}, 75^{t h}$, and $98^{t h}$ percentiles. 

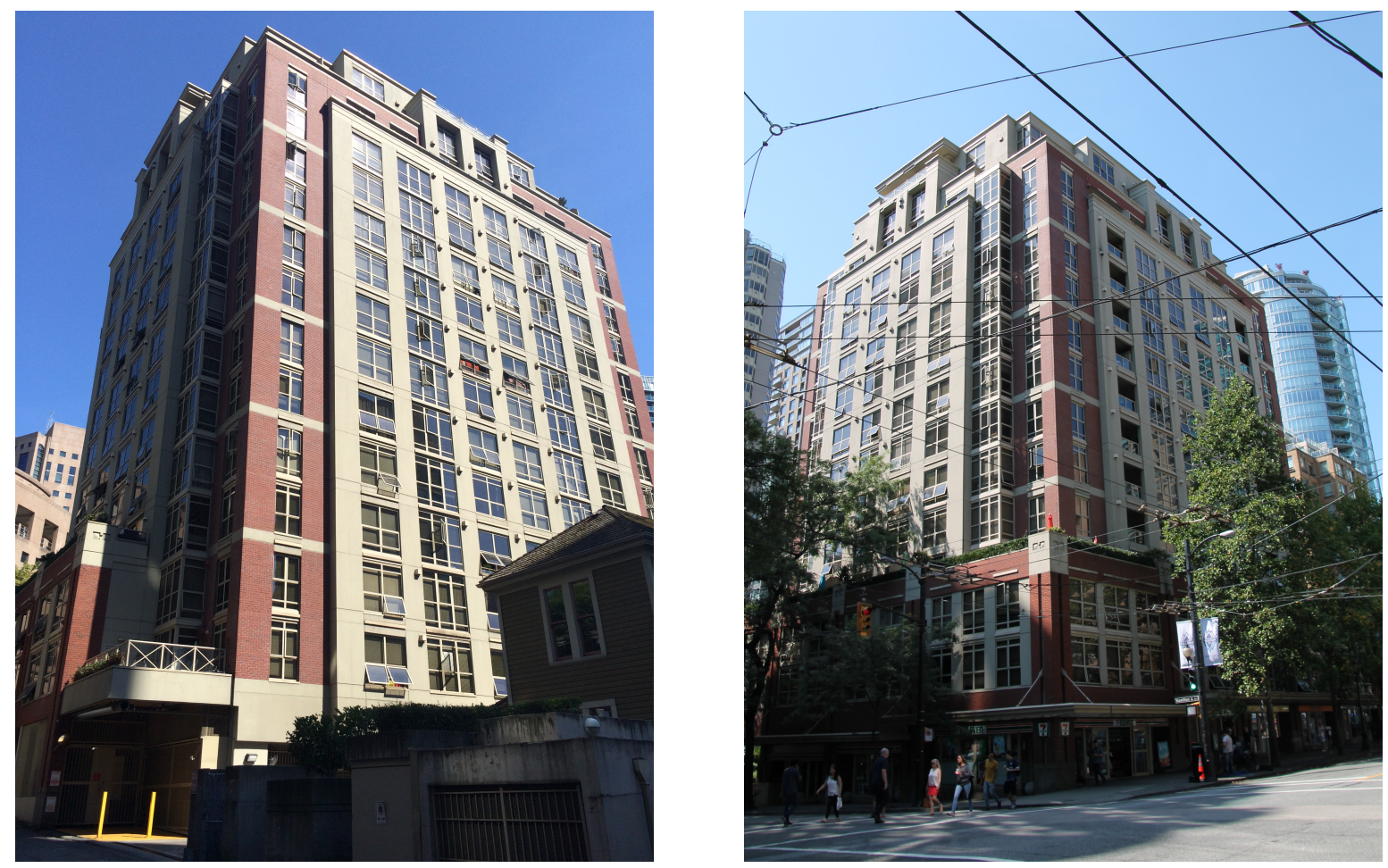

Figure 13: Case 2 - The Heritage Court Building from two directions

\subsubsection{Results and Discussion}

There should be three stable modes at approximately $1.22,1.28$, and $1.45 \mathrm{~Hz}$ in the applied frequency range [1]. In the stability diagram, three stable modes stand out as vertical lines for both the original and modified correlation function matrix, see Fig. 14. The cluster plot in Fig. 15 illustrates the same tendency by three clusters of estimates. Both Fig. 14 and Fig. 15 contain all identified modal parameters without any validation of the estimates.

The estimated modal parameters from the original correlation function matrix, however, drift slightly as the number of block rows of the Hankel matrix changes whereas the estimates of the modified correlation function matrix are more stable, see Fig. 14. In the cluster plot, the clusters of stable modes are denser and shift when we apply the algorithm to reduce the noise tail, see Fig. 15, whereas the damping estimates for the original correlation function matrix are unstable and form scattered clusters.

Since we extract six modes for each model order, there are an additional three modes for each identification as seen in Figs. 14 and 15. These modes are spurious and instable regardless of the correlation function matrix applied in this identification process. Furthermore, some of these spurious modes have negative damping. The spurious modes from the original correlation function matrix have natural frequencies closer to the three stable modes whereas the natural frequencies of the spurious modes from the modified correlation function reside below.

Based on this study, reducing the noise tail creates more stable estimates that are less dependent on the model order (number of block rows). This indicates that the reduction of statistical errors is essential for a more stable and reliable estimation of the modal parameters using operational modal analysis.

\section{Conclusion}

The finite length of the measured vibrations introduces system dependent statistical errors in operational modal analysis. These errors cause random errors in the estimated correlation function matrix and bias errors 


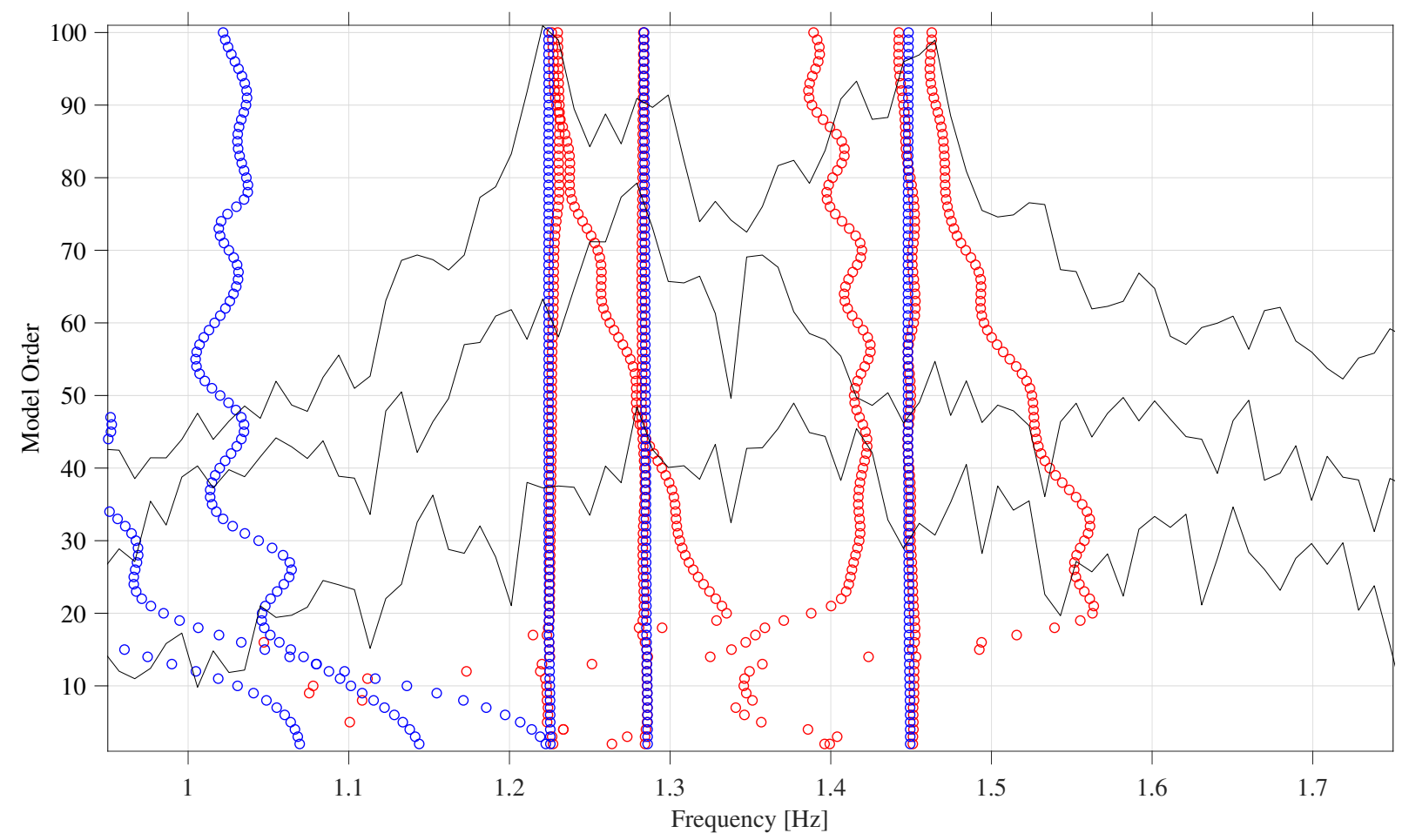

Figure 14: Case 2 - Heritage Court Tower, data set 1 [1]: Stability diagram based on the original correlation function matrix (black) and the modified correlation function matrix (blue), both including 500 time lags and disregarding the first ten time lags 


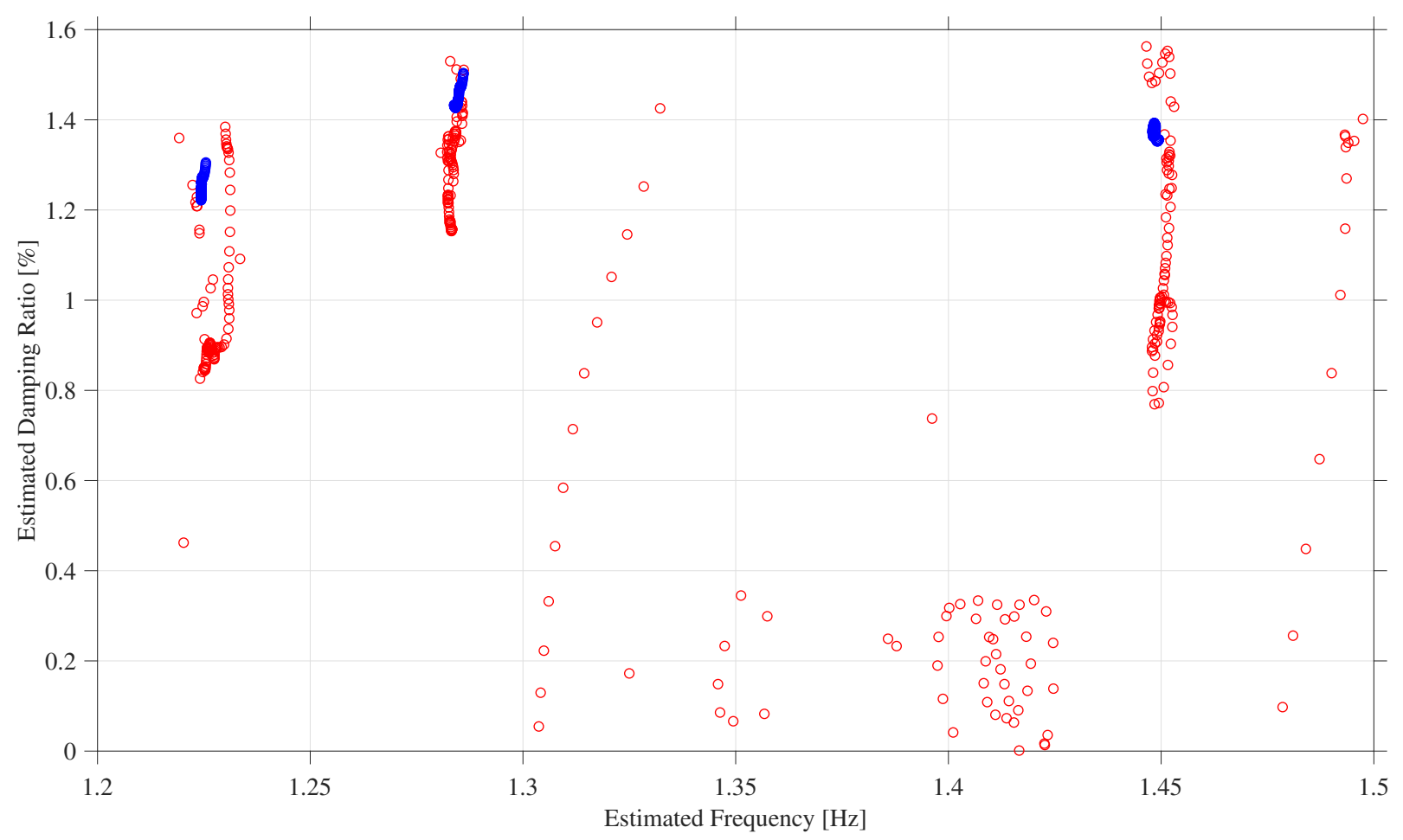

Figure 15: Case 2 - Heritage Court Tower, data set 1 [1]: Cluster plot with the natural frequency and damping ratio for estimated modes from the stability diagram based on the original correlation function matrix (black) and the modified correlation function matrix (blue), both including 500 time lags and disregarding the first ten time lags 
in the envelopes of the matrix. The noise tail (erratic behaviour in the tail region of the correlation function matrix) indicates high levels of bias errors. Furthermore, these statistical errors transfer to an identification process of the modal parameters resulting in estimated modal parameters with random and bias errors. Since the bias error relates to the envelope of the correlation function, it mainly affects the damping estimates. Hence, in operational modal analysis, it is essential to reduce the noise tail so we decrease the bias error on damping estimates.

In this article, we introduced an automatic algorithm to reduce the noise tail in the estimated correlation function matrix. The algorithm decorrelates the estimated correlation function matrix through a condensation technique, auto-regression models, and a poly-reference technique. It detects the noise tail of the decorrelated auto-correlation functions by fitting two lines to the Hilbert envelope. Then it modifies the envelope of each noise tail to mimic the physical part using the regression fit. The algorithm reduces the bias error of the statistical errors but random errors still remain in the correlation function matrix.

Based on the studies presented in this article, the statistical errors make identification techniques biassed and unstable since the techniques fit a correlation function matrix with both random and bias errors which increase with increasing number of time lags. The number of included time lags of the correlation function matrix and the model order of the identification technique affect this fit and often cause instability - here weakly-excited modes seem to be more unstable. The algorithm reduces the effect of statistical errors on a correlation function matrix. Thereby, it increases the stability and reliability and it reduces the bias error in the identification process of the modal parameters.

\section{Acknowledgements}

The authors acknowledge the funding received from the Centre for Oil and Gas - DTU/Danish Hydrocarbon Research and Technology Centre (DHRTC).

The authors thank Michael Vigs $\varnothing$ and Thomas Kabel from Aarhus University for their helpful discussions.

\section{Appendix A. Automated Modal Decorrelation of Estimated Correlation Function}

We use the condensation technique in [12] to decorrelate and equalise the energy for each component in the estimated correlation function matrix. It is a dimensionality reduction based on the Principle Component Analysis using the Singular Value Decomposition [14]. We perform a singular value decomposition on the estimated correlation function matrix at time lag zero.

$$
\mathbf{U S U}^{T}=\operatorname{SVD}\left(\widetilde{\mathbf{R}}_{y}(0)\right)
$$

where $\mathbf{U}$ is a unitary matrix holding the singular vectors, $\left[\mathbf{U}_{1}, \mathbf{U}_{2}, \ldots, \mathbf{U}_{n}\right]$, and $\mathbf{S}$ is a diagonal matrix that holds the descending singular values, $\sigma_{1}>\sigma_{2}>\cdots>\sigma_{n} \geq 0$.

$$
\mathbf{S}=\left[\begin{array}{cccc}
\sigma_{1} & 0 & \ldots & 0 \\
0 & \sigma_{2} & \ldots & 0 \\
\vdots & \vdots & \ddots & \vdots \\
0 & 0 & \ldots & \sigma_{n}
\end{array}\right]
$$

To reduce the dimension, we remove the lower singular values in $\mathbf{S}$ and the corresponding singular vectors in $\mathbf{U}$ and we set up a transformation matrix, $\mathbf{T}$. In this algorithm, the dimentional reduction is optional and it is mainly applied to reduce the computation time.

$$
\mathbf{T}=\mathbf{U}_{m} \mathbf{S}_{m}^{\frac{1}{2}}
$$

where $\mathbf{S}_{m}$ is the upper left $m \times m$ block of the singular value matrix, and $\mathbf{U}_{m}$ contains the first $m$ singular vectors. Some identification techniques apply similar dimensionality reduction using the singular value 
decomposition. These techniques apply this approach to help stabilise the identification process of modal parameters. This implementation of the singular value decomposition has a different objective: We want to create a new correlation function matrix with reduced noise tails that we can use with any identification technique that utilise correlation function matrices. In order to achieve this, we need to decorrelate the estimated correlation function matrix and equalise the energy for each modal auto-correlation function.

This transformation matrix, $\mathbf{T}$, decorrelates and condenses the correlation function matrix to $m$ components.

$$
\mathbf{Y}(k)=\mathbf{T}^{\dagger} \widetilde{\mathbf{R}}_{y}(k)\left(\mathbf{T}^{\dagger}\right)^{\top}
$$

Since the singular vectors are, by definition, orthogonal, it creates some problems when we have a correlation between modes. In such a case, the transformation matrix, Eq. (A.3), is orthogonal and insufficient to decorrelate the correlation function matrix since the mode shapes of the system are non-orthogonal. Therefore, we modify this transformation to decorrelate systems that are more complex. We use a similar approach as [17] to estimate eigenvectors using auto-regression models and a poly reference technique. First, we set up two block Hankel matrices using Eq. (A.4).

$$
\begin{aligned}
\mathbf{H}_{1} & =\left[\begin{array}{lllll}
\mathbf{Y}(1)^{\top} & \mathbf{Y}(2)^{\top} & \mathbf{Y}(3)^{\top} & \ldots & \mathbf{Y}(K-2)^{\top} \\
\mathbf{Y}(2)^{\top} & \mathbf{Y}(3)^{\top} & \mathbf{Y}(4)^{\top} & \ldots & \mathbf{Y}(K-1)^{\top}
\end{array}\right] \\
\mathbf{H}_{2} & =\left[\begin{array}{lllll}
\mathbf{Y}(3)^{\top} & \mathbf{Y}(4)^{\top} & \mathbf{Y}(5)^{\top} & \ldots & \mathbf{Y}(K)^{\top}
\end{array}\right]
\end{aligned}
$$

We estimate the auto-regression matrices by the use of the block Hankel matrices.

$$
\left[\begin{array}{ll}
\widehat{\mathbf{A}}_{2} & \widehat{\mathbf{A}}_{1}
\end{array}\right]=\mathbf{H}_{2} \mathbf{H}_{1}^{\dagger}
$$

Then we form the companion matrix.

$$
\mathbf{A}_{c}=\left[\begin{array}{cc}
\mathbf{0} & \mathbf{I} \\
\widehat{\mathbf{A}}_{2} & \widehat{\mathbf{A}}_{1}
\end{array}\right]
$$

We perform an eigenvalue decomposition of the companion matrix and obtain the eigenvectors, V. Then we modify the transformation matrix from Eq. (A.3).

$$
\mathbf{Z}=\mathbf{T V}
$$

Finally, we decorrelate the estimated correlation function matrix into an approximated modal correlation function matrix, which we will call the decorrelated correlation function matrix.

$$
\widehat{\mathbf{R}}_{q}(\tau)=\mathbf{Z}^{\dagger} \widetilde{\mathbf{R}}_{y}(\tau)\left(\mathbf{Z}^{\dagger}\right)^{\top}
$$

On the diagonals of this matrix, the correlation functions are decorrelated to auto-correlation functions, which corresponds to free decays of SDOF systems. Since this is an approximated decorrelation, the decorrelated correlation function matrix is a non-diagonal matrix.

\section{References}

[1] R. Brincker, C. Ventura, Introduction to operational modal analysis, John Wiley and Sons, Inc., Chichester, West Sussex,UK, 2015.

[2] G. C. James, T. G. Carne, J. P. Lauffer, The natural excitation technique (next) for modal parameter extraction, The International Journal of Analytical and Experimental Modal Analysis 10 (4) (1995) 260-277.

[3] R. Brincker, On the application of correlation function matrices in oma, Mechanical Systems and Signal Processing 87 (2017) 17-22. doi:10.1016/j.ymssp.2016.08.009.

[4] J. S. Bendat, A. G. Piersol, Random data: analysis and measurement procedures, 4th Edition, Wiley-Blackwell, Hoboken, N.J., USA, 2010.

[5] M. Tarp $\varnothing$, T. Friis, C. Georgakis, R. Brincker, The statistical errors in the estimated correlation function matrix for operational modal analysis, Submitted to Journal of Sound and Vibration. 
[6] E. Orlowitz, A. Brandt, Influence of correlation estimation methods on damping estimates, 5th International Operational Modal Analysis Conference.

[7] L. Giampellegrini, Uncertainty in correlation-driven operational modal analysis, Ph.D. thesis, University College London, UK, 64-74 (2007).

[8] A. Bajrić, J. Høgsberg, F. Rüdinger, Evaluation of damping estimates by automated operational modal analysis for offshore wind turbine tower vibrations, Renewable Energy 116 (2018) 153 - 163, real-time monitoring, prognosis and resilient control for wind energy systems. doi:https://doi.org/10.1016/j.renene.2017.03.043. URL http://www.sciencedirect.com/science/article/pii/S0960148117302355

[9] M. Tarp $\varnothing$, P. Olsen, S. Amador, M. Juul, R. Brincker, On minimizing the influence of the noise tail of correlation functions in operational modal analysis (2017). doi:https://doi.org/10.1016/j.proeng.2017.09.282.

URL http://www.sciencedirect.com/science/article/pii/S1877705817337633

[10] M. Tarp $\varnothing$, P. Olsen, M. Juul, S. Amador, T. Friis, R. Brincker, Statistical error reduction for correlation-driven operational modal analysis, Proceedings of ISMA 2018.

[11] B. A. Pridham, J. C. Wilson, A study of damping errors in correlation-driven stochastic realizations using short data sets, Probabilistic Engineering Mechanics 18 (1) (2003) 61 - 77. doi:https://doi.org/10.1016/S0266-8920(02)00042-5. URL http://www.sciencedirect.com/science/article/pii/S0266892002000425

[12] P. Olsen, M. Juul, R. Brincker, Condensation of the correlation functions in modal testing, Mechanical Systems and Signal Processing 118 (2019) 377 - 387. doi:https://doi.org/10.1016/j.ymssp.2018.08.036. URL http://www.sciencedirect.com/science/article/pii/S0888327018305752

[13] J. N. Juang, R. S. Pappa, An eigensystem realization algorithm for modal parameter identification and model reduction, Journal of Guidance 8 (5) (1985) 620-627.

[14] I. Jolliffe, Principal component analysis, 2nd Edition, Springer Verlag, New York, USA, 2002.

[15] E. Orlowitz, A. Brandt, Influence of noise in correlation function estimates for operational modal analysis, Topics in Modal Analysis \& Testing, Proceedings of the 36th IMAC 9 (2019) 55-64. doi:https://doi.org/10.1007/978-3-319-74700-2.

[16] A. Brandt, Noise and vibration analysis : signal analysis and experimental procedures, 1st Edition, Wiley, Chichester, UK, 2011.

[17] H. Vold, J. Kundrat, G. Rocklin, R. Russell, A multi-input modal estimation algorithm for mini-computers, SAE Technical Paper 820194 (1982) 10doi:https://doi.org/10.4271/820194.

[18] R. G. Lyons, Understanding digital signal processing, 3rd Edition, Prentice Hall, Upper Saddle River, N.J.,USA, 2011.

[19] C. L. Lawson, R. J. Hanson, Solving least squares problems, Prentice-Hall, Englewood Cliffs, USA, 1974.

[20] P. C. Hansen, V. Pereyra, G. Scherer, Least squares data fitting with applications, Johns Hopkins University Press, Baltimore, MD, USA, 2013

[21] F. N. Fritsch, R. E. Carlson, Monotone piecewise cubic interpolation, SIAM Journal on Numerical Analysis 17 (1980) 238-246. doi:https://doi.org/10.1137/0717021.

URL http://www.sciencedirect.com/science/article/pii/S1877705817339656

[22] S. R. Ibrahim, E. C. Mikulcik, A method for the direct identification of vibration parameters from the free response, The Shock and Vibration Inform. Ctr. Shock and Vibration Bull. (1977) 183-198. 